\title{
ON THE RING OF HOLOMORPHIC FUNCTIONS ON AN OPEN RIEMANN SURFACE $\left(^{1}\right)$
}

\author{
BY \\ IRWIN KRA
}

1. Introduction and summary of results. It is well known (Bers [1], Nakai [5]) that an open Riemann surface is determined up to mirror image by its ring of holomorphic functions. Thus, it is quite natural to expect that one can find an algebraic characterization of the ring of holomorphic functions on an open Riemann surface. Also, one expects that all the information about the conformal structure of an open Riemann surface can be recovered from the ring of holomorphic or the field of meromorphic functions on it. The purpose of this paper is to investigate the relationships between the conformal structure of an open Riemann surface and the algebraic structure of the ring of holomorphic functions on it. Our main result (Theorem III) characterizes conformal structure in terms of simple algebraictopological concepts. In $\S 5$ we obtain a purely algebraic characterization of certain rings of holomorphic functions. It is not as simple or as elegant as one would like. Alternate algebraic characterization of rings of holomorphic and fields of meromorphic functions are discussed in §6. One of the characterizations is due to the referee. The hypotheses used in the referee's characterization are simpler and more natural than those appearing in any of the other algebraic characterizations discussed in this paper. The author thanks the referee for pointing out this alternate approach and for the various suggestions which have simplified the presentation of this paper.

Any nontrivial holomorphic mapping $F: X \rightarrow Y$ between two open Riemann surfaces induces a $C$-monomorphism $F^{\prime}: H(Y) \rightarrow H(X)$ between their rings of holomorphic functions defined as follows:

$$
\left(F^{\prime} f\right)(x)=f(F(x)), \quad f \in H(Y), \quad x \in X .
$$

Essentially these are the only nontrivial morphisms between rings of holomorphic functions on open Riemann surfaces. We obtain

Received by the editors May 10, 1966 and, in revised form, March 3, 1967.

( ${ }^{1}$ ) This paper is part of the author's doctoral dissertation at Columbia University, prepared under the supervision of Professor Lipman Bers, while the author was supported by an NSF Graduate Fellowship. Preparation of this paper was partially supported by the Air Force Office of Scientific Research, Office of Aerospace Research, United States Air Force, under AFOSR Grant No. 335-63. 
THEOREM I. Let $X$ and $Y$ be open Riemann surfaces with rings of holomorphic functions $H(X)$ and $H(Y)$ respectively. Let $F: H(Y) \rightarrow H(X)$ be any ring homomorphism such that

(1) $F$ restricted to the field of constants $C$ is an automorphism $\sigma$ of $C$ which fixes $(-1)^{1 / 2}$, and

(2) the image of $F$ contains at least one nonconstant function.

Then

(1) $F$ is a monomorphism,

(2) $\sigma$ is the identity map, and

(3) there is a unique holomorphic mapping $F^{*}: X \rightarrow Y$ which induces $F$ (that is $\left.(F f)(x)=f\left(F^{*}(x)\right), f \in H(Y), x \in X\right)$.

Clearly the requirement that $\sigma\left((-1)^{1 / 2}\right)=(-1)^{1 / 2}$ is a normalization condition. Under any automorphism of $C,(-1)^{1 / 2}$ is either fixed or sent into $-(-1)^{1 / 2}$. An analogous result holds under this assumption. Theorem $I$ is a generalization of theorems of Bers [1], Nakai [5], and Royden [9].

A similar theorem is proven for homomorphisms between fields of meromorphic functions on arbitrary Riemann surfaces. We then obtain the following algebraic characterization of surfaces of finite genus.

THEOREM II. Let $X$ be a Riemann surface with field of meromorphic functions $K(X)$. Then $X$ is conformally equivalent to a domain of a closed surface if and only if $K(X)$ contains a field $C$-isomorphic to an algebraic function field in one variable which separates the points of $X$.

The property "separation of points" can be characterized algebraically. The points of $X$ are in one-to-one correspondence with the places of $K(X)$ whose value group is the group of integers. The value of a function at a point is the value of the corresponding place at the function. (See Iss'sa [3].)

A characterization of rings of holomorphic functions on an open Riemann surface is obtained in

THEOREM III. Let $X$ be a connected, locally compact, Hausdorff space, and $C(X)$ the ring of complex-valued continuous functions on $X$. Let $A$ be a subalgebra of $C(X)$. Assume that

(1) A separates points and contains the constants,

(2) every nonconstant function in $A$ is an open mapping, and

(3) for every $x \in X$, the ideal $M(x)=\{f \in A ; f(x)=0\}$ is principal.

Then $X$ can be given a unique conformal structure which respects the topology such that every $f \in A$ becomes a holomorphic function on $X$. In particular $X$ is an open Riemann surface. Furthermore, $A$ is the algebra of all holomorphic functions on $X$ if and only if $A$ is maximal among subalgebras of $C(X)$ that satisfy conditions (1) to (3). 
(A mapping $f: X \rightarrow Y$ is called open if for every open set $U$ in $X, f(U)$ is open in $Y$.)

2. Homomorphisms between rings of holomorphic functions. In this section $X$, $Y$, and $Z$ always represent open Riemann surfaces. The ring of holomorphic functions on the open Riemann surface $X$ is denoted by $H(X)$.

Lemma (2.1). Let $F: H(X) \rightarrow C$ be any $C$-algebra homomorphism, then there is a unique point $x \in X$ such that $F(f)=f(x)$, for $f \in H(X)$.

Proof. See Royden [9].

Proof of Theorem I. Since this theorem follows very closely the results of Bers [1] and Nakai [5], we only outline the proof.

(1) For each $x \in X$ and $f \in H(Y)$, let $\theta_{x}(f)$ be defined to be $(F f)(x)$. Then $\sigma^{-1} \circ \theta_{x}$ is a $C$-algebra homomorphism of $H(Y)$ into $C$. By Lemma 2.1 there is a unique point $F^{*}(x)$ in $Y$ such that $\sigma^{-1}((F f)(x))=f\left(F^{*}(x)\right)$; that is, $(F f)(x)$ $=\sigma\left(f\left(F^{*}(x)\right)\right)$.

(2) If $\left\{y_{n}\right\}=\left\{F^{*}\left(x_{n}\right)\right\}$ is an infinite, distinct, and discrete set of points in $Y$, then by the generalized Mittag-Leffler theorem (see, for example, Florack [2]), there is a function $f \in H(Y)$ such that $f\left(y_{n}\right)=n$. Thus

$$
(F f)\left(x_{n}\right)=\sigma\left(f\left(F^{*}\left(x_{n}\right)\right)\right)=\sigma\left(f\left(y_{n}\right)\right)=\sigma(n)=n ;
$$

that is, $\left\{x_{n}\right\}$ is discrete in $X$.

(3) As an immediate consequence of (2) we obtain that the closure of $F^{*}(K)$ is compact in $Y$ whenever $K$ is compact in $X$.

(4) Given $\beta \in C$, there is an $f \in H(Y)$ and $x \in X$ such that

(i) $(F f)(x)=\beta$, and

(ii) $F f$ is univalent in a neighborhood of $x$.

We have assumed that there is an $f_{0} \in H(Y)$ such that $F f_{0} \notin C$. Thus there is an $x \in X$ and a neighborhood of $x$ in which $F f_{0}$ is univalent. If $\left(F f_{0}\right)(x)=\alpha$, then $f=f_{0}+\sigma^{-1}(\beta-\alpha)$ has the required properties.

(5) The automorphism $\sigma$ is the identity. Since $\sigma$ is the identity on $Q\left[(-1)^{1 / 2}\right]$ (the rationals with $(-1)^{1 / 2}$ adjoined), it suffices to show that $\sigma$ is continuous with respect to the usual topology on $C$. But $\sigma$ is additive; thus it is enough to show that it is bounded on a neighborhood of the origin. Choose $f \in H(Y)$ and $x \in X$ such that $(F f)(x)=0$ and $F f$ is univalent in a neighborhood $U$ of $x$. We may assume that the closure of $U(\mathrm{Cl} U)$ is compact. Since $(F f)(U)$ is open and $0 \in(F f)(U)$, there is a $\delta>0$ such that $|\alpha|<\delta$ implies $\alpha \in(F f)(U)$. If $\sigma^{-1}$ is not bounded on a neighborhood of the origin, we can choose a sequence $\alpha_{n} \rightarrow 0$ for which $\sigma^{-1}\left(\alpha_{n}\right) \rightarrow \infty$. We may assume $\left|\alpha_{n}\right|<\delta$ for all $n$. Thus there is a point $x_{n} \in U$ such that $(F f)\left(x_{n}\right)=\alpha_{n}$. If $y_{n}=F^{*}\left(x_{n}\right)$, then the sequence $\left\{y_{n}\right\}$ is contained in the compact set $\mathrm{Cl}\left(F^{*}(\mathrm{Cl} U)\right)$. Thus $f\left(y_{n}\right)$ is bounded. But we have the contradiction

$$
f\left(y_{n}\right)=f\left(F^{*}\left(x_{n}\right)\right)=\sigma^{-1}\left((F f)\left(x_{n}\right)\right)=\sigma^{-1}\left(\alpha_{n}\right) \rightarrow \infty .
$$


(6) Standard arguments (which may be found in Royden's paper [9], for example) now show that $F^{*}$ is continuous, holomorphic, and induces $F$.

Definition (2.2). Let $X, Y$ be open Riemann surfaces with rings of holomorphic functions $H(X)$ and $H(Y)$ respectively. Then a homomorphism $F: H(Y)$ $\rightarrow H(X)$ is called admissible if $F$ sends constants onto constants and if the image of $F$ contains at least one nonconstant function. $F$ is called direct if $F\left((-1)^{1 / 2}\right)$ $=(-1)^{1 / 2}$, and indirect otherwise.

Theorem I describes the direct admissible homomorphisms. Each indirect homomorphism $F$ is induced by an antiholomorphic map $F^{*}$ in the sense that $(F f)(x)$ is the complex conjugate of $f\left(F^{*}(x)\right)$.

Corollary 1 (Functorial Properties of ${ }^{*}$ ). (a) Let $F: H(X) \rightarrow H(X)$ be the identity mapping; then $F^{*}: X \rightarrow X$ is also the identity mapping.

(b) If $F: H(Y) \rightarrow H(X)$ and $G: H(Z) \rightarrow H(Y)$ are admissible, then so is $F \circ G$ and $(F \circ G)^{*}=G^{*} \circ F^{*}$.

Corollary 2 (Bers [1], NAKai [5]). Let $X$ and $Y$ be open Riemann surfaces with rings of holomorphic functions $H(X)$ and $H(Y)$ respectively. Let $F: H(Y) \rightarrow H(X)$ be any ring isomorphism. Then there is a unique homeomorphism $F^{*}: X \rightarrow Y$ which induces $F$. The map $F^{*}$ is conformal if $F\left((-1)^{1 / 2}\right)=(-1)^{1 / 2}$, and anticonformal otherwise.

Proof. By uniqueness of $F^{*}$, it suffices to show that $F$ is admissible. For then $F^{*}$ and $F^{-1 *}$ will be inverse mappings. Since $F$ is an isomorphism, it suffices to show that $F$ preserves constants. This follows from the fact that a function $f \in H(Y)$ is constant if and only if for each $\alpha \in Q\left[(-1)^{1 / 2}\right]$ there is a $g \in H(Y)$ such that $f-\alpha=g^{2}$.

\section{An algebraic characterization of Riemann surfaces of finite genus.}

THEOREM (3.1). Let $X$ and $Y$ be Riemann surfaces with fields of meromorphic functions $K(X)$ and $K(Y)$ respectively. Let $F: K(Y) \rightarrow K(X)$ be a homomorphism mapping constants onto constants. Assume $F\left((-1)^{1 / 2}\right)=(-1)^{1 / 2}$. In addition, if $Y$ is closed assume that $F(\lambda)=\lambda$ for $\lambda \in C$. Then there is a unique holomorphic mapping $F^{*}: X \rightarrow Y$ which induces $F$ (that is, $\left.(F f)(x)=f\left(F^{*}(x)\right), f \in K(Y), x \in X\right)$.

Proof. Assume $Y$ is open. Iss'sa [3] has shown that every discrete valuation of rank 1 on $K(Y)$ is equivalent to evaluation of orders at a unique point of $Y$. Thus $F$ preserves holomorphic functions. Since $F$ is a monomorphism, it is admissible. Because every meromorphic function is a ratio of holomorphic functions, the theorem follows from Theorem I.

For $Y$ closed the theorem is well known. We sketch a proof. Let $x \in X$. Define $v_{x}(f)=$ order of $f$ at $x$ for $f \in K(X)$. Then $v_{x} \circ F$ is a valuation on $K(Y)$, and is thus given by a unique point $F^{*}(x) \in Y$. Arguments similar to those used in the proof of Theorem I show that $F^{*}$ is holomorphic and induces $F$. 
For $Y$ closed the assumption that the homomorphism $F$ is the identity on the field of constants is essential. Let $Y=C \cup\{\infty\}$ and $X=C$. If $\sigma$ is any automorphism of $\boldsymbol{C}$, then it extends trivially to $\boldsymbol{C}(z)$, the field of rational functions. Clearly $\sigma: K(Y) \rightarrow K(X)$ cannot be induced by any continuous map of $X$ into $Y$ whenever $\sigma$ is not continuous. It is apparently well known that "almost all" compact Riemann surfaces of the same genus have isomorphic function fields. To the best of the author's knowledge, the only place to find this result in print is in his doctoral dissertation [4].

Proof of Theorem II. Assume there exists a holomorphic injection $F: X \rightarrow Y$ with $Y$ closed. As usual, define the adjoint $F^{\prime}: K(Y) \rightarrow K(X)$ by $F^{\prime} f=f \circ F$ for $f \in K(Y)$. Then $F^{\prime}$ is a $C$-monomorphism. It remains to show that $F^{\prime}(K(Y))$ separates points on $X$. Now $x, y \in X$ and $x \neq y$ implies that $F(x) \neq F(y)$. Choose $f \in K(Y)$ such that $f(F(x)) \neq f(F(y))$. Thus $\left(F^{\prime} f\right)(x) \neq\left(F^{\prime} f\right)(y)$.

Conversely, assume $R \subset K(X)$ and $R$ is $C$-isomorphic to $K(Y)$ with $Y$ closed. Thus there is a surjective $C$-isomorphism $F: K(Y) \rightarrow R$. By Theorem 3.1 there is a unique holomorphic mapping $F^{*}: X \rightarrow Y$ such that $F f=f \circ F^{*}$ for $f \in K(Y)$. We must show that $F^{*}$ is one-to-one. Assume there are points $x, y \in X$ with $x \neq y$ and $F^{*}(x)=F^{*}(y)$. Since $R$ separates points, we can choose $f \in R$ such that $f(x) \neq f(y)$. Because $F$ is surjective, $f=F g$ with $g \in K(Y)$. We thus have that $F(g(x)) \neq F(g(y))$, and $F(g(x))=g\left(F^{*}(x)\right)=g\left(F^{*}(y)\right)=F(g(y))$. We have arrived at an obvious contradiction.

The assumption that the subfield of $K(X)$ separates the points of $X$ cannot be dropped. Let $X$ be a double covering of the punctured unit disk $\{z \in C ; 0<|z|<1\}$ with ramification at $z=1 / 2,1 / 3, \ldots$ Then $C(z) \subset K(X)$. The surface $X$ is of infinite genus. Clearly $C(z)$ does not separate the points of $X$.

4. An algebraic-topological characterization of the ring of holomorphic functions. In this section we prove Theorem III. Proposition 4.2 is a direct by-product of the work required to prove our main theorem.

Definition (4.1). Let $A$ be an algebra of continuous complex-valued functions on a topological space $X$ which contains the constants. We say that $A$ is regular at $x \in X$ if there is a compact set $K$ with $x \notin K$ such that for each $f$ in $A|f(x)| \leqq$ $\sup \{|f(y)| ; y \in K\}$.

An element $t \in A$ is called a uniformizer at $x$ if

(1) the maximal ideal $M(x)=\{f \in A ; f(x)=0\}$ is the principal ideal generated by $t$, and

(2) $\bigcap_{n=1}^{\infty} M(x)^{n}=\{0\}$.

The assumption that $t$ is a uniformizer at $x$ will enable us to express every $f \in A$ as a formal power series in $t$. (Note that in Theorem III we do not assume condition (2). The stronger topological assumptions of the theorem make such a hypothesis unnecessary. We will point out in the sequel the places where (2) is needed.) Regularity at $x$ states that $A$ satisfies a maximum modulus principle, and this will guarantee the convergence of the formal power series. We will obtain 
Proposition (4.2). Let $A$ be an algebra of continuous complex-valued functions on a topological space $X$ which contains the constants and separates points. Assume there is a point $x \in X$ and a function $t \in A$ such that $t$ is $a$ uniformizer at $x$ and $A$ is regular at $x$. Then (1) there is a $C$-algebra monomorphism of $A$ into the algebra of functions analytic on the closed unit disc, and (2) there exists a Riemann surface $X^{\prime}$ and an algebra $A^{\prime}$ which is $C$-isomorphic to $A$ such that $X^{\prime}$ is proper for $A^{\prime}$. The pair $\left(A^{\prime}, X^{\prime}\right)$ is unique up to a conformal equivalence.

Proof. ( $A^{\prime}$ is proper for $X^{\prime}$ means that the field of fractions of $A^{\prime}$ separates the points of $X^{\prime}$, and $X^{\prime}$ is the largest surface for which $A^{\prime}$ has this property. $\left(A^{\prime}, X^{\prime}\right)$ is conformally equivalent to $\left(A^{\prime \prime}, X^{\prime \prime}\right)$ if there is a conformal homeomorphism $\tau$ of $X^{\prime}$ onto $X^{\prime \prime}$ such that the adjoint of $\tau$ maps $A^{\prime \prime}$ onto $A^{\prime}$.) Statement (2) is a direct consequence of (1) and Proposition 2 of Royden's paper [10]. Statement (1) follows from Lemmas 4.7 and 4.9. The following lemmas and definitions assume the hypothesis of this proposition.

Lemma (4.3). (a) Let $x \in X$. Then $\theta(f)=f(x)$ for $f \in A$ is a C-algebra homomorphism onto $C$ with kernel $M(x)$. In particular, $A / M(x) \cong C$.

(b) Let $t$ be a uniformizer at $x \in X$, and let $y \in X$. Then $t(y)=0$ if and only if $y=x$.

Proof. Statement (a) is obvious. Statement (b) follows easily since $M(x)$ is a maximal ideal, and $A$ separates points.

We next show that we may cancel uniformizers in an equation.

Lemma (4.4). Let $t$ be a uniformizer at $x$. Suppose $f, g \in A$ and $f t=g t$, then $f=g$.

Proof. If $y \neq x$, then $t(y) \neq 0$, and thus $f(y)=g(y)$. If $x$ is not isolated (as in hypothesis of Theorem III) then by continuity $f(x)=g(x)$. In the general case, assume $(f-g)(x)=\alpha \neq 0$, and let $h=-\alpha^{-1}(f-g-\alpha)$. Now $h \in A$, and $h(x)=0$, $h(y)=1$ for $y \neq x$. We have $h \in M(X)$ and since $h^{n}=h, h \in M(x)^{n}$ for all integers $n$. Thus by definition of uniformizer $h=0$. This contradicts the assumption that $h(y) \neq 0$ for $y \neq x$.

Definition (4.5). Let $t \in A$ be a uniformizer at $x \in X$. Let $f \in A$, we say that the formal power series $\sum_{i=0}^{\infty} a_{i} t^{i}$, with $a_{i} \in C$, is associated with $f$, and write $f^{\prime \prime}=" \sum_{i=0}^{\infty} a_{i} t^{i}$ if $\left(f-\sum_{i=0}^{k} a_{i} t^{i}\right) / t^{k} \in M(x)$ for $k=0,1,2, \ldots$

Lemma (4.6). Let $t \in A$ be a uniformizer at $x \in X$. Then for every $f$ in $A$, there is $a$ unique formal power series in $t$ associated with $f$.

Proof. Let $a_{0}=f(x)$. Then $a_{0}$ is the unique complex number such that $f-a_{0} \in M(x)$. Having chosen $a_{0}, \ldots, a_{n}$ uniquely, we show that $a_{n+1}$ is uniquely determined. By the induction assumption

$$
g_{n}=\left(f-\sum_{i=0}^{n} a_{i} t^{i}\right) / t^{n} \in M(x)
$$


Thus there is a unique $h_{n} \in A$ such that $g_{n}=t h_{n}$. Let $h_{n}(x)=a_{n+1}$. Then $a_{n+1}$ is the unique complex number such that $h_{n}-a_{n+1} \in M(x)$. But

$$
\frac{f-\sum_{i=0}^{n+1} a_{i} t^{i}}{t^{n+1}}=\frac{f-\sum_{i=0}^{n} a_{i} t^{i}-a_{n+1} t^{n+1}}{t^{n+1}}=h_{n}-a_{n+1},
$$

which also shows that $a_{n+1}$ is unique.

Lemma (4.7). Let $t \in A$ be a uniformizer at $x \in X$. Then the map which sends $f \in A$ into the formal power series in $t$ associated with $f$ is $a$ C-algebra monomorphism into the ring of formal power series in $t$ over $C$.

Proof. Standard arguments show that the map is a $C$-homomorphism. It is one-to-one since $f^{\prime \prime}=" 0$ implies $f / t^{k} \in M(x)$ for $k=0,1,2, \ldots$ Hence $f \in M(x)^{k}$ for $k=1,2, \ldots$, and thus $f=0$ (we used condition (2)).

Lemma (4.8). Let $t \in A$ be a uniformizer at $x \in X$. Define $T f=(f-f(x)) / t$ for $f \in A$. Then $T$ is a linear operator in $A$ whose kernel is $C$. Furthermore if we let $a_{n}=\left(T^{n} f\right)(x)$, then

and $f^{\prime \prime}=" \sum_{i=0}^{\infty} a_{i} t^{i}$.

$$
T^{n} f=\left(f-\sum_{i=0}^{n-1} a_{i} t^{i}\right) / t^{n}
$$

Proof. Clearly $f-f(x) \in M(x)$. Thus we can find a unique $g \in A$ such that $f-f(x)$ $=g t$. Hence $T$ is a well-defined operator in $A$. Linearity is clear. Also, it is obvious that $C=$ kernel $T$. To deduce the formula for $T^{n}$ we use induction on $n$. The formula holds for $n=1$. Assume we have formula for $n \geqq 1$. Then

$$
T^{n+1} f=T\left(T^{n} f\right)=\frac{T^{n} f-\left(T^{n} f\right)(x)}{t}=\frac{\left(f-\sum_{i=0}^{n-1} a_{i} t^{i}\right) / t^{n}-a_{n}}{t}=\frac{f-\sum_{i=0}^{n} a_{i} t^{i}}{t^{n+1}} .
$$

To prove that $f^{\prime \prime}=" \sum_{i=0}^{\infty} a_{i} t^{i}$, it suffices to show, by uniqueness of the associated power series, that $\left(f-\sum_{i=0}^{n} a_{i} t^{i}\right) / t^{n} \in M(x)$. But $\left(f-\sum_{i=0}^{n} a_{i} t^{i}\right) / t^{n}=T^{n} f-\left(T^{n} f\right)(x)$ for $n=0,1,2, \ldots$

Lemma (4.9). Let $t \in A$ be a uniformizer at $x \in X$. Assume that $A$ is regular at $x$. Then for every $f \in A$, its associated power series in thas a positive radius of convergence which is independent of $f$.

Proof. There is a compact set $K$ with $x \notin K$, and $|f(x)| \leqq \sup \{|f(y)| ; y \in K\}$ for all $f \in A$. For $f \in A$, define $\|f\|=\sup \{|f(y)| ; y \in K\}$. With respect to this seminorm the operator $T$ of the previous lemma is bounded. For

$$
\|T f\|=\sup \{|(f(y)-f(x)) / t(y)| ; y \in K\},
$$

and $t \neq 0$ on $K$. Since $K$ is compact and $t$ is continuous, there is an $\varepsilon>0$ such that 
$|t| \geqq \varepsilon$ on $K$. Hence $\|T f\| \leqq 2\|f\| \varepsilon^{-1}$, or $\|T\| \leqq 2 \varepsilon^{-1}$. If $f^{\prime \prime}=" \sum_{i=0}^{\infty} a_{i} t^{i}$, then $a_{i}=\left(T^{i} f\right)(x)$. And

$$
\left|a_{i}\right| \leqq\left\|T^{i} f\right\| \leqq\|T\|^{i}\|f\| \leqq 2^{i} \varepsilon^{-i}\|f\| .
$$

The radius of convergence of the power series in $t$ associated with $f$ is thus at least $\varepsilon / 2$.

COROLlaRY OF PROOF. If $f \neq 0$, then $\|f\| \neq 0$.

Proof. If $\|f\|=0$, then $a_{n}=0$ for all $n$. Thus $f=0$ by Lemma 4.7.

We have completed the proof of Proposition 4.2. The next lemma is needed in the proof of Theorem III.

LEMMA (4.10). Let $f$ be a continuous open mapping from a topological space $X$ into $C$, then $f$ satisfies the maximum modulus principle. Furthermore for any open set $U$ with compact closure

$$
\sup \{|f(x)| ; x \in \mathrm{Cl} U\}=\sup \{|f(x)| ; x \in \mathrm{Cl} U-U\} .
$$

In particular, if $A$ is an algebra of complex-valued continuous functions on a locally compact space $X$, and $A$ consists only of open or constant functions, then $A$ is regular at every point of $X$.

Proof. Choose an open set $U$ in $X$. Assume there is an $x \in U$ such that $|f(x)|$ $\geqq|f(y)|$ for all $y \in U$. Since $f(U)$ is an open set containing $f(x)$, there is an $\varepsilon>0$ such that the $\varepsilon$ ball about $f(x)$ is contained in $f(U)$. This contradicts the maximality of $f$ at $x$. If $\mathrm{Cl} U$ is compact, then $f$ assumes a maximum on $\mathrm{Cl} U$. This maximum cannot be assumed on $U$, hence it must be assumed on $\mathrm{Cl} U-U$. The validity of the last part of the lemma is now obvious.

Proof of Theorem III. Let $x \in X$, and let $t \in A$ be a generator of the principal ideal $M(x)$. Let $f^{\prime \prime}=" \sum_{i=0}^{\infty} a_{i} t^{i}$. (Recall that condition (2) in the definition of a uniformizer was not needed except in the proof of Lemma 4.7.) Let $U$ be any relatively compact neighborhood of $x$. Let $\varepsilon=\inf \{|t(y)| ; y \in \mathrm{Cl} U-U\}$. Then $\varepsilon>0$, and the formal power series in $t$ associated with $f$ converges for $|t| \leqq \varepsilon / 2$. We now show that there is an open neighborhood $V$ of $x$ in which this power series actually represents the function $f$. Let $V=\{y \in U ;|t(y)|<\varepsilon / 4\}$. Then $V$ is a nonempty open set. Now, using the notation and results of Lemmas 4.8 and 4.9 we have that

Thus,

$$
f(y)-\sum_{i=0}^{n} a_{i} t^{i}(y)=t^{n+1}(y)\left(T^{n+1} f\right)(y)
$$

$$
\left|f(y)-\sum_{i=0}^{n} a_{i} t^{i}(y)\right| \leqq(1 / 2)^{n+1}\|f\|, \quad \text { for } y \in V,
$$

where we compute the norm of $f$ on the set $U$. The function $t$ must be one-to-one in $V$. For $t\left(x_{1}\right)=t\left(x_{2}\right)$ with $x_{1}, x_{2} \in V$ implies $f\left(x_{1}\right)=f\left(x_{2}\right)$ for all $f \in A$. This is impossible since $A$ separates points. The function $t$ is a local parameter in $V$, 
because it is a one-to-one, open, continuous mapping onto an open neighborhood of the origin in $C$. The elements of $A$ are clearly holomorphic functions of the local parameters. Thus $X$ is a Riemann surface, and $A$ is an algebra of holomorphic functions on $X$. The conformal structure obtained in this manner is clearly the only one in which the algebra $A$ becomes an algebra of holomorphic functions, since a single nonconstant holomorphic function is enough to determine the conformal structure of a Riemann surface. The proof of the first part of the theorem is complete.

Let $H(X)$ be the algebra of all holomorphic functions on the Riemann surface $X$. Then it is well known that $H(X)$ satisfies conditions (1) through (3) of the theorem. If $H(X) \subset A^{\prime} \subset C(X)$, with $A^{\prime}$ satisfying these conditions, then by the first part of the theorem $X$ can be given a conformal structure in which the algebra $A^{\prime}$ becomes an algebra of holomorphic functions. Thus every function in $A^{\prime}$ is a holomorphic function of the local parameter in terms of which the conformal structure is defined. A function $f \in A^{\prime}$ is a local parameter at $x \in X$ if and only if $f$ is one-to-one in a neighborhood of $x$. For every $x \in X$, we can find a local parameter for $x$ in the algebra $H(X)$. Thus every function $f \in A^{\prime}$ is locally a holomorphic function of elements of $H(X)$. Thus $A^{\prime} \subset H(X)$.

Conversely, a maximal subalgebra $A$ of $C(X)$ satisfying conditions (1) through (3) defines a conformal structure on $X$ for which $A=H(X)$.

Remarks. (1) The assumption that $A$ separates points can be weakened. It suffices to assume that $A$ locally separates points.

(2) In general, the open mapping assumption cannot be dropped. The algebra of complex-valued real-analytic functions on the real line satisfies all the other assumptions of the theorem.

(3) If we assume that $X$ is a surface, then the open mapping assumption may be replaced by the assumption that the algebra $A$ satisfies a maximum modulus principle. The proof that the generators of the principal maximal ideals give local parameters uses Invariance of Domain.

5. Towards an algebraic characterization of rings of holomorphic functions. In this section we obtain an algebraic characterization of certain rings of holomorphic functions on open Riemann surfaces. The characterization (see condition (C)) is not as simple nor as elegant as one would like.

Let $R$ be a commutative $C$-algebra with identity. (Henceforth "algebra" means "commutative $C$-algebra with identity".) We are interested in algebras $R$ that satisfy:

(A) The kernel of every $C$-algebra homomorphism of $R$ (onto $C$ ) is a principal ideal and

(B) $\bigcap\{M(\theta) ; \theta \in S\}=\{0\}$ where $M(\theta)=$ Kernel $\theta$ and $S=\operatorname{Hom}_{C}(R, C)=$ set of $C$-homomorphisms of $R$.

We call $S$ the structure space of $R$. Let $F(S)$ be the set of complex-valued functions 
on $S$. If $f \in R$, define $f^{\wedge} \in F(S)$ by $f^{\wedge}(\theta)=\theta(f)$ for $\theta \in S$. Then $\wedge: R \rightarrow F(S)$ is a $C$-algebra monomorphism, whose image is denoted by $R^{\wedge}$. We topologize $S$ by making $f^{\wedge}$ a continuous function on $S$ for all $f \in R$. It is easy to see that $S$ is now a Hausdorff topological space. If $\theta_{0} \in S$, then sets of the form

$$
U\left(\theta_{0}\right)=U\left(\theta_{0} ; f_{1}, \ldots, f_{n} ; \varepsilon\right)=\left\{\theta \in S ;\left|\theta\left(f_{i}\right)\right|<\varepsilon, i=1, \ldots, n\right\}
$$

with $\left\{f_{1}, \ldots, f_{n}\right\} \subset M\left(\theta_{0}\right), \varepsilon>0$, and $n$ a positive integer, form a basis for the open neighborhoods of $\theta_{0}$.

The ring $R^{\wedge}$ is now an algebra of complex-valued continuous functions on $S$. If $t_{0}$ is the generator of the principal ideal $M\left(\theta_{0}\right)$ with $\theta_{0} \in S$, then $t_{0}(\theta)=0$ if and only if $\theta=\theta_{0}$. Thus functions in $R^{\wedge}$ vanishing at a point of $S$ form a principal ideal. The space $S$ is not yet locally compact, nor are the nonconstant functions in $R^{\wedge}$ open mappings. We impose one more condition on the ring $R$ :

(C) (1) For every $\theta_{0} \in S$, and all sets $\left\{f_{1}, \ldots, f_{n}\right\} \subset M\left(\theta_{0}\right)-\{0\}$, and all $\varepsilon>0$, there is a $\delta>0$ such that whenever $|\alpha|<\delta$, we can find an element $\theta \in S$ with $\theta\left(f_{1}\right)=\alpha$ and $\left|\theta\left(f_{i}\right)\right|<\varepsilon$ for $i=2, \ldots, n$.

(2) Furthermore, if $f_{1}$ generates the principal ideal $M\left(\theta_{0}\right)$, then there is a set $\left\{f_{2}, \ldots, f_{n}\right\} \subset M\left(\theta_{0}\right)$, and $\varepsilon>0$, and a $\delta>0$ such that the element $\theta$ whose existence is guaranteed by (1) is unique.

Definition (5.1). A ring (that is a $C$-algebra and) that satisfies conditions (A), (B) and $(\mathrm{C})$ is called a holomorphic ring.

The ring of holomorphic functions on an open Riemann surface is a holomorphic ring. We have previously remarked that condition (A) is satisfied (because of Lemma 2.1 and the generalized Weierstrass theorem). Condition (B) states that the ring of holomorphic functions separates points. To verify (C) one needs to know that holomorphic functions are open mappings and that the topology of an open Riemann surface is the weakest topology making all the holomorphic functions continuous. This last fact is a consequence of the theorem originally stated by Remmert [7] and proved by Narasimhan [6] which asserts that any open Riemann surface has a one-to-one, regular, proper holomorphic imbedding into $C^{3}$. Because of the Remmert theorem, we could let $n=3$ in condition (C). However, this assumption does not simplify the arguments that follow. We will hence not use it. Our task now is to show that holomorphic rings deserve their name.

Lemma (5.2). Let $R$ be a holomorphic ring with structure space $S$. Let $f \in R-C$, then $f^{\wedge}$ is an open mapping.

Proof. It suffices to show that $f^{\wedge}(U)$ is open in $C$, where $U$ is an open set in a basis for the topology for $S$. We may thus assume that $U=U\left(\theta_{0} ; f_{1}, \ldots, f_{n} ; \varepsilon\right)$ with $\varepsilon>0$ and $f_{i} \in$ kernel $\theta_{0}$ for $i=1, \ldots, n$. Let $\alpha \in f^{\wedge}(U)$, and choose $\theta_{1} \in U$ such that $f^{\wedge}\left(\theta_{1}\right)=\alpha$. If $\varepsilon_{i}=\theta_{1}\left(f_{i}\right)$, then $\left|\varepsilon_{i}\right|<\varepsilon$ because $\theta_{1} \in U$. Now,

$$
\left\{f-\alpha, f_{1}-\varepsilon_{1}, \ldots, f_{n}-\varepsilon_{n}\right\} \subset \text { kernel } \theta_{1},
$$


and $f-\alpha \neq 0$ since $f \notin C$. If $\eta=\min \left\{\varepsilon-\left|\varepsilon_{i}\right|, i=1, \ldots, n\right\}$, then $\eta>0$. By condition (C), there is a $\delta>0$ such that

$$
\theta(f-\alpha)=\beta \text { with }|\beta|<\delta
$$

and $\left|\theta\left(f_{i}-\varepsilon_{\imath}\right)\right|<\eta$ for $i=1, \ldots, n$ has a solution for some $\theta \in S$. We next show that $\theta \in U$. We have that

$$
\begin{aligned}
\left|\theta\left(f_{i}\right)\right| & =\left|\theta\left(f_{i}-\varepsilon_{i}+\varepsilon_{i}\right)\right|=\left|\theta\left(f_{i}-\varepsilon_{i}\right)+\varepsilon_{i}\right| \\
& \leqq\left|\theta\left(f_{i}-\varepsilon_{i}\right)\right|+\left|\varepsilon_{i}\right|<\eta+\left|\varepsilon_{i}\right| \leqq \varepsilon-\left|\varepsilon_{i}\right|+\left|\varepsilon_{i}\right|=\varepsilon .
\end{aligned}
$$

Thus the $\delta$-ball about $\alpha$ is contained in $f^{\wedge}(U)$.

LemMa (5.3). Let $R$ be a holomorphic ring with structure space $S$. Then for each $\theta_{0} \in S$, there is an open neighborhood $D$ of $\theta_{0}$ that is homeomorphic to the open unit disc. In particular, $S$ is locally compact.

Proof. Let $f_{1}$ be a generator of the principal ideal $M\left(\theta_{0}\right)$. Choose $\left\{f_{2}, \ldots, f_{n}\right\}$, $\varepsilon>0, \delta>0$, such that for all $\alpha \in C$ with $|\alpha|<\delta$,

$$
\theta\left(f_{1}\right)=\alpha \text { with }\left|\theta\left(f_{i}\right)\right|<\varepsilon \text { for } i=2, \ldots, n
$$

has a unique solution $\theta \in S$.

Let $D=U\left(\theta_{0} ; f_{1} ; \delta\right) \cap U\left(\theta_{0} ; f_{2}, \ldots, f_{n} ; \varepsilon\right)$. Then $D$ is open in $S$, and $f_{1} \mid D$ is a homeomorphism onto $\{z \in C ;|z|<\delta\}$.

We have thus obtained the following "algebraic" version of Theorem III.

THEOREM (5.4). Let $R$ be a holomorphic ring with structure space $S$. Let $X$ be a connected component of $S$. Let $A$ be the ring $R^{\wedge}$ restricted to $X$. Then $X$ can be given a unique conformal structure such that $A$ becomes an algebra of holomorphic functions on the Riemann surface $X$. Furthermore, the map which restricts a function in $R^{\wedge}$ to $X$ is a $C$-algebra isomorphism.

The next theorem gives some information about the components of the structure space of a holomorphic ring. We need two lemmas.

LEMMA (5.5). Let $R$ be a holomorphic ring with structure space $S$. Let $\theta \in S$, and let $\theta_{n} \in S$ for $n=1,2, \ldots$ Then $\theta_{n} \rightarrow \theta$ if and only if $f^{\wedge}\left(\theta_{n}\right) \rightarrow f^{\wedge}(\theta)$ for all $f \in R$.

Proof. The function $f^{\wedge}$, with $f \in R$, is continuous on $S$. Thus $\theta_{n} \rightarrow \theta$ implies that $f^{\wedge}\left(\theta_{n}\right) \rightarrow f^{\wedge}(\theta)$. To prove the converse, assume that $\theta_{n}$ does not converge to $\theta$. Then there is a neighborhood $U$ of $\theta$ such that for every integer $n_{0}$, we can find an integer $n \geqq n_{0}$ with $\theta_{n} \notin U$. We may assume $U=U\left(\theta ; f_{1}, \ldots, f_{m} ; \varepsilon\right)$ with $\left\{f_{1}, \ldots, f_{m}\right\} \subset$ kernel $\theta$ and $\varepsilon>0$. Choose $n_{1} \geqq 1$ such that $\theta_{n_{1}} \notin U$. Having chosen $n_{1}<n_{2}<\cdots<n_{k}$, with $\theta_{n_{i}} \notin U$ for $i=1, \ldots, k$, choose $n_{k+1} \geqq n_{k}+1$ such that $\theta_{n_{k+1}} \notin U$. Thus $\left|f_{j}\left(\theta_{n_{i}}\right)\right| \geqq \varepsilon$ for some $j \in\{i, \ldots, m\}=J$. Because $J$ is a finite set there is at least one $j_{0} \in J$ such that $\left|\hat{j_{0}}\left(\theta_{n_{i}}\right)\right| \geqq \varepsilon$ for an infinite set of the $n_{i}$. This contradicts the fact that $f_{j_{0}}(\theta)=0$. 
LeMma (5.6). Let $R$ be a holomorphic ring with structure space $S$. Let $\left\{\theta_{n}\right\}$ be a discrete sequence in $S$. Then there is an $f \in R$ such that $\lim _{n \rightarrow \infty} f^{\wedge}\left(\theta_{n}\right)$ does not exist.

Proof. Assume otherwise. For $f \in R$, define $\theta(f)=\lim _{n \rightarrow \infty} f^{\wedge}\left(\theta_{n}\right)$. Then $\theta$ is a $C$-homomorphism of $R$. Thus $\theta \in S$, and $\theta(f)=f^{\wedge}(\theta)$ for all $f \in R$. By the previous lemma $\theta_{n} \rightarrow \theta$. We have arrived at an obvious contradiction.

THEOREM (5.7). Let $R$ be a holomorphic ring with structure space $S$. Let $X$ be a connected component of $S$. Let $A$ be the ring $R^{\wedge}$ restricted to $X$. Then $A$ cannot be extended to a ring of holomorphic functions on any Riemann surface $Y$ which properly contains $X$.

Proof. This theorem is a direct consequence of Lemma 5.6.

THEOREM (5.8). Let $R_{i}(i=1,2)$ be a holomorphic ring with structure space $S_{i}$. Let $F: R_{2} \rightarrow R_{1}$ be a $C$-algebra homomorphism. Then there exists a unique holomorphic mapping $F^{*}: S_{1} \rightarrow S_{2}$ which induces $F$ (that is $\theta(F f)=\left(F^{*} \theta\right)(f), f \in R_{2}$, $\left.\theta \in S_{1}\right)$.

Proof. The proof uses only Lemma 5.6 and standard arguments. It is left to the reader.

COROLlARY. If $F$ is an isomorphism, then $F^{*}$ is a homeomorphism.

REMARKS. (1) The following example shows that the structure space of a holomorphic ring need not be connected. Let $R$ be the ring of those rational functions with poles only on the unit circle. Then it is easy to see that the structure space $S$ of $R$ is the complement in $C$ of the unit circle. $S$ has "the usual" conformal structure.

(2) Each of the following guarantees that the structure space $S$ of a holomorphic ring $R$ consists of a single component:

(a) There exists a component $X$ such that $R^{\wedge} \mid X$ is the ring of all holomorphic functions on $X$.

(b) There is a component $X$ such that $f \in R^{\wedge}$, and $1 / f$ holomorphic on $X$ implies that $1 / f \in R^{\wedge}$.

Conditions involving only the elements of $R$ that guarantee the connectedness of $S$ are not known to the author.

(3) A holomorphic ring is always an integral domain. Thus a holomorphic ring cannot be the ring of all holomorphic functions on its structure space when the latter is not connected. It is fairly easy to modify the definition of holomorphic ring to allow for this more general case.

6. On alternate approaches. We outline very briefly alternate approaches to the problem of the last section.

Definition (6.1). Let $R$ be an integral domain and a $C$-algebra whose field of fractions is $K$. We call $K$ a meromorphic field if it satisfies conditions $\left(\mathrm{A}^{\prime}\right),\left(\mathrm{B}^{\prime}\right)$ and $\left(\mathrm{C}^{\prime}\right)$. 
(A') Every $C$-valued place of $K$ over $C$ whose valuation ring contains $R$ is the localization of $R$ at some maximal principal ideal of $R$.

Let $S=$ set of $C$-valued places of $K$ over $C$ whose valuation rings contain $R$. As before, $S$ is called the structure space of $K$. If $\theta \in S$, denote by $V(\theta)$, the valuation ring of $\theta$, and by $M(\theta)$, its valuation ideal.

(B') $\bigcap\{V(\theta) ; \theta \in S\}=R$, and $\bigcap\{M(\theta) ; \theta \in S\}=\{0\}$. We endow $S$ with the weakest topology which makes every $f \in K$ a continuous function $f^{\wedge}: S \rightarrow C \cup\{\infty\}$, where $f^{\wedge}(\theta)=\theta(f)$ for $f \in R, \theta \in S$. We thus obtain the field $K^{\wedge}=\left\{f^{\wedge} ; f \in K\right\}$.

$\left(\mathrm{C}^{\prime}\right)$ (1) For every $\theta_{0} \in S$, and all $\left\{f_{1}, f_{2}\right\} \subset M\left(\theta_{0}\right) \cap R-\{0\}$, and all $\varepsilon>0$, there is a $\delta>0$ such that $|\alpha|<\delta$ implies we can find an element $\theta \in S$ with $\theta\left(f_{1}\right)=\alpha$ and $\left|\theta\left(f_{2}\right)\right|<\varepsilon$.

(2) For every $\theta_{0} \in S$, there is a neighborhood $U$ of $\theta_{0}$ such that $f^{\wedge}$ is bounded on $U$ for all $f \in R$.

It turns out that the field of meromorphic functions on an open Riemann surface is a meromorphic field and conversely:

THEOREM (6.2). Let $K$ be a meromorphic field with structure space $S$. Then $S$ can be given a unique conformal structure such that for every $f \in K$, the element $f^{\wedge}$ is a meromorphic function on $S$. In particular, every component of $S$ is an open Riemann surface.

The proof of Theorem 6.2 is similar to the proof of Theorem 5.4. Condition $\left(C^{\prime} 1\right)$ which is clearly equivalent to $(\mathrm{Cl})$ is used to prove that the elements of $R^{\wedge}-C$ (where $R^{\wedge}$ is the image of $R$ in $K^{\wedge}$ ) are open mappings. For each $x \in K$, we form a copy $\Sigma_{x}$ of the compact sphere $C \cup\{\infty\}$. Let $P=\prod_{x \in K} \Sigma_{x}$ with the weak product topology. Then by the Tychonoff theorem $P$ is a compact Hausdorff space. The structure space $S$ of $K$ is naturally imbedded into $P$. It is not a closed subset of $P$. However, the closure in $P$ of a set of places of $K$ consists only of places. Thus condition ( $\left.\mathrm{C}^{\prime} 2\right)$ implies that $S$ is locally compact. Now this theorem follows from Theorem III.

Other, essentially different, algebraic (algebraic-topological) characterizations of rings of holomorphic functions on an open Riemann surface will be found in a forthcoming paper of Richards [8].

The referee has pointed out that the following hypothesis can substitute for condition (C):

(C*) Given $\theta_{0} \in S$, there is a $K \subset S$ with $\theta_{0} \notin K$ such that (1) for each $f$ in $R$ we have $\left|\theta_{0}(f)\right| \leqq \sup \{|\theta(f)| ; \theta \in K\}<\infty$, and (2) there is an $f$ in $R$ with $\left|\theta_{0}(f)\right|<$ $\inf \{|\theta(f)| ; \theta \in K\}$.

To prove that a ring $R$ that satisfies conditions (A), (B), and $\left(\mathrm{C}^{*}\right)$ can be realized as a ring of holomorphic functions on its structure space $S$, we may proceed as follows: Let $\theta_{0} \in S$, and let $t$ generate the principal ideal $M\left(\theta_{0}\right)=\left\{f \in R ; \theta_{0}(f)=0\right\}$.

(1) If $\{f, g\} \subset R$ and $f^{\wedge} t^{\wedge}=g^{\wedge} t^{\wedge}$, then $f=g$. 
(2) If $\varepsilon=\inf \{|\theta(t)| ; \theta \in K\}$, then $\varepsilon>0$ ( $K$ is the set whose properties are described by hypothesis $\left(\mathrm{C}^{*}\right)$ ).

(3) If $f \in R$, then the formal power series in $t^{\wedge}$ associated with $f^{\wedge}$ has a radius of convergence $\rho \geqq \varepsilon / 2$.

(4) For $\alpha \in C,|\alpha|<\rho$, the map $t \rightarrow \alpha$ has a natural extension to a homomorphism $\theta_{\alpha}$ of $R$ onto $C$.

(5) As a basis for an open neighborhood system of $\theta_{0} \in S$, we take sets of the form $U(\delta)=\left\{\theta_{\alpha} ;|\alpha|<\delta\right\}$ with $0<\delta<\rho$. The function $t^{\wedge}$ is then a uniformizer at $\theta_{0}$.

(6) With the above topology and set of local uniformizers, each component of $S$ becomes a Riemann surface on which $R^{\wedge}$ acts as an algebra of holomorphic functions.

It should be noted that steps (5) and (6) of the above procedure are similar to the way Royden endows the set of all primitive local represe itations of an algebra with a one-dimensional complex analytic structure [10, Proposition 1].

\section{REFERENCES}

1. L. Bers, On rings of analytic functions, Bull. Amer. Math. Soc. 54 (1948), 311-315.

2. H. Florack, Reguläre und meromorphe Funktionen auf nicht geschlossenen Riemannschen Flächen, Schr. Math. Inst. Univ. Münster 1 (1948).

3. H. Iss'sa, On the meromorphic function field of a Stein variety, Ann. of Math. (2) 83 (1966), 34-46.

4. I. Kra, Conformal structure and algebraic structure, Doctoral dissertation, Columbia Univ., New York, 1966.

5. M. Nakai, On rings of analytic functions on Riemann surfaces, Proc. Japan. Acad. 39 (1963), 79-84.

6. R. Narasimhan, Imbedding of open Riemann surfaces, Nachr. Acad. Wiss. Göttingen Math.-Phys. Kl. II 7 (1960), 159-165.

7. R. Remmert, Sur les espaces analytiques holomorphiquement séparables et holomorphiquement convexes, C. R. Acad. Sci. Paris 243 (1956), 118-121.

8. I. Richards, A criterion for rings of analytic functions, Trans. Amer. Math. Soc. 128 (1967), 523-530.

9. H. L. Royden, Rings of analytic and meromorphic functions, Trans. Amer. Math. Soc. 83 (1956), 269-276.

10. - Algebras of bounded analytic functions on Riemann surfaces, Acta Math. 114 (1965), 113-142.

Columbia University, New York, New York

Massachusetts Institute of Technology, Cambridge, Massachusetts 